\title{
Skyrmion-(Anti)Vortex Coupling in a Chiral Magnet-Superconductor Heterostructure
}

\author{
A. P. Petrović $\odot,{ }^{1, *}$ M. Raju $\odot,{ }^{1, \dagger}$ X. Y. Tee, ${ }^{1}$ A. Louat $\odot,{ }^{2}$ I. Maggio-Aprile $\odot,{ }^{3}$ R. M. Menezes $\odot,{ }^{4,5}$ \\ M. J. Wyszyński®, ${ }^{4}$ N. K. Duong, ${ }^{1}$ M. Reznikov $\odot,{ }^{2}$ Ch. Renner $\odot,{ }^{3}$ M. V. Milošević $\odot,{ }^{4}$ and C. Panagopoulos $\oplus^{1, \$}$ \\ ${ }^{1}$ Division of Physics and Applied Physics, School of Physical and Mathematical Sciences, \\ Nanyang Technological University, 637371 Singapore \\ ${ }^{2}$ Department of Physics, Technion, Haifa 32000, Israel \\ ${ }^{3}$ Department of Quantum Matter Physics, Université de Genève, 24 Quai Ernest Ansermet, CH-1211 Geneva 4, Switzerland \\ ${ }^{4}$ Department of Physics, University of Antwerp, Groenenborgerlaan 171, B-2020 Antwerp, Belgium \\ ${ }^{5}$ Departamento de Física, Universidade Federal de Pernambuco, Cidade Universitária, 50670-901 Recife-PE, Brazil
}

(Received 8 November 2020; accepted 14 January 2021; published 17 March 2021)

\begin{abstract}
We report experimental coupling of chiral magnetism and superconductivity in $[\operatorname{IrFeCoPt}] / \mathrm{Nb}$ heterostructures. The stray field of skyrmions with radius $\approx 50 \mathrm{~nm}$ is sufficient to nucleate antivortices in a $25 \mathrm{~nm} \mathrm{Nb}$ film, with unique signatures in the magnetization, critical current, and flux dynamics, corroborated via simulations. We also detect a thermally tunable Rashba-Edelstein exchange coupling in the isolated skyrmion phase. This realization of a strongly interacting skyrmion-(anti)vortex system opens a path toward controllable topological hybrid materials, unattainable to date.
\end{abstract}

DOI: 10.1103/PhysRevLett.126.117205

Introduction.-Chiral magnets and superconductors host topological excitations known as skyrmions and vortices, whose duality was recognized in the 1980s [1]. Recently, skyrmion-vortex pairing in heterostructures [2-6] has been proposed as a method of coupling chiral magnetism and superconductivity: a combination anticipated to deliver novel hybrid behavior $[7,8]$. For example, one can envisage changing the superconducting order parameter symmetry by imprinting noncollinear exchange fields from skyrmions or spin helices onto Cooper pairs. Such fields are gauge equivalent to a Zeeman field combined with spin-orbit coupling (SOC) and may hence create a topological superconductor hosting Majorana fermions at its boundaries and vortex cores [9-16]. Controlling the nucleation and dynamics of vortices in the presence of skyrmions is the key to unlocking the potential of chiral magnetsuperconductor hybrids for topological quantum computation [17,18] and fluxonics [19].

Skyrmions and vortices interact via two mechanisms. The first originates from the Rashba-Edelstein effect [20]: the skyrmion exchange field combines with interfacial SOC to induce circulating spin-polarized supercurrents, which interfere with vortex currents [2,3,21-23]. This interaction ("exchange coupling") requires contact between superconductor and magnet, and depends on the sign and

Published by the American Physical Society under the terms of the Creative Commons Attribution 4.0 International license. Further distribution of this work must maintain attribution to the author(s) and the published article's title, journal citation, and DOI. magnitude of the SOC and exchange field. The second mechanism - stray field coupling — allows skyrmion-vortex interaction without electronic contact at distances greater than the exchange length [4]. The sign and magnitude of this interaction are determined by the current profile induced in the superconductor, which depends on the magnetic layer thickness $d_{m}$, skyrmion chirality, and skyrmion-vortex separation [24]. The skyrmion core polarization is antiparallel to the applied magnetic field $H$ and hence repels vortices. However, a sufficiently large skyrmion can nucleate an antivortex in a nearby superconductor, creating a bound pair of topological solitons experimentally unexplored to date.

These interactions can be modulated by adjusting the skyrmion/vortex length scales. Stray field coupling is enhanced by increasing skyrmion size, whereas exchange coupling requires Rashba-Edelstein and (anti)vortex currents to circulate with similar radii. This corresponds to the condition $\xi<r_{\mathrm{sk}}<\lambda$ [Fig. 1(a)] [3], where $\xi, \lambda$ are the superconducting coherence and penetration lengths, and $r_{\mathrm{sk}}$ is the skyrmion radius. Minimizing $H$ (and hence the vortex density) also favors antivortex formation-but aside from $\mathrm{Co} / \mathrm{Ru}(0001)$ monolayers [25], stabilizing skyrmions at low temperature in thin films has typically required $H \gtrsim 1 \mathrm{~T}[26,27]$. This exceeds the upper critical field $H_{c 2}$ of many $s$-wave superconductors, precluding skyrmion-(anti)vortex coupling. Here we present the first chiral magnet-superconductor heterostructures to host stable skyrmions at low fields and temperatures below the superconducting transition $T_{c}$. Experiments and simulations both indicate that skyrmion stray fields create antivortices in the superconductor, strongly coupling spin and flux topologies. We also detect signatures of 


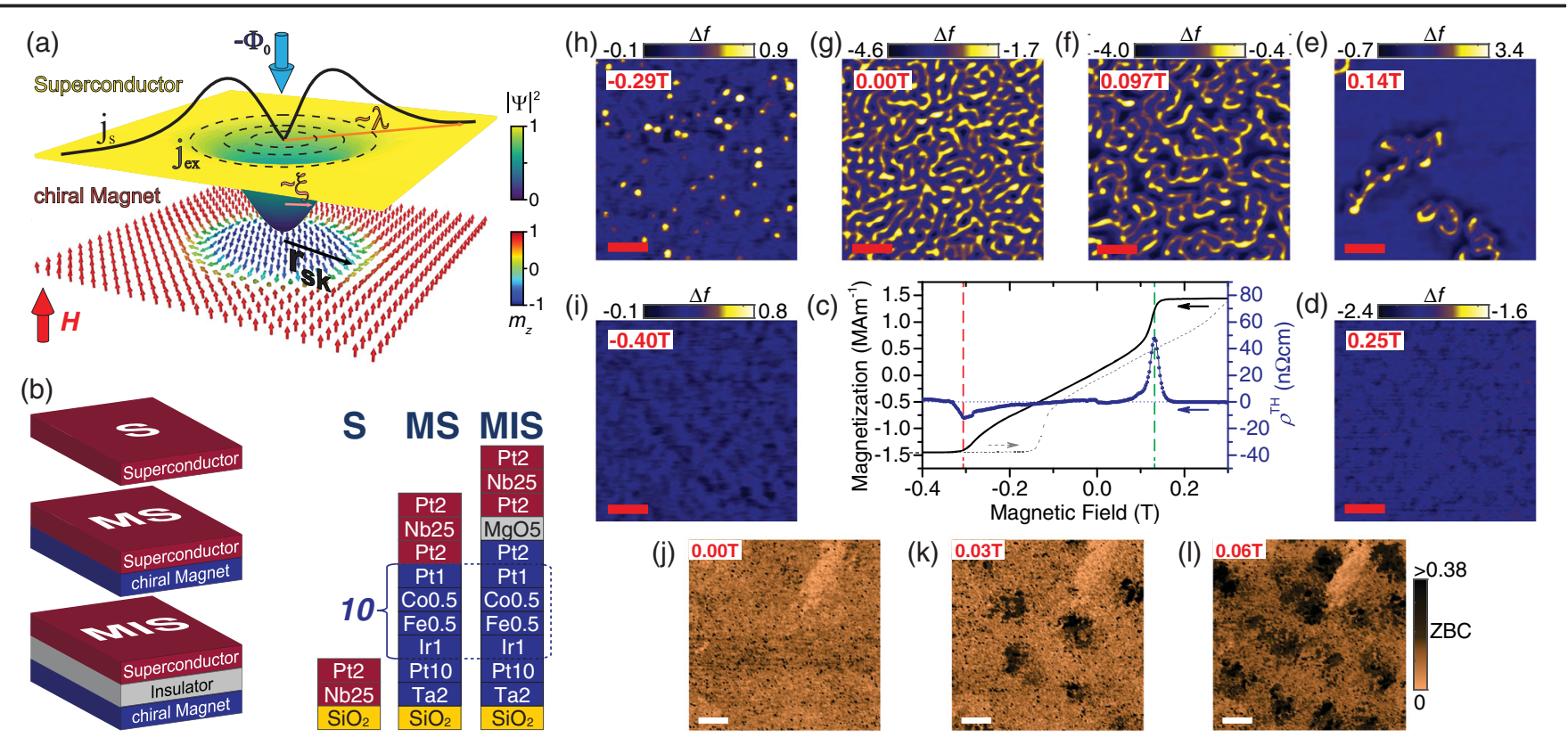

FIG. 1. (a) Schematic of a Néel skyrmion creating an antivortex with flux $-\Phi_{0} \equiv-h / 2 e$ antiparallel to the external magnetic field $H$. Antivortex currents $j_{s}$ flow at radii up to $\lambda$; Rashba-Edelstein currents $j_{\mathrm{ex}}$ are maximal at $r_{\mathrm{sk}}$, where the local out-of-plane magnetic moment $m_{z}=0$. The superconducting order parameter $|\Psi|$ is suppressed over a length $\xi$ in the vortex core. (b) Sample compositions: numbers (e.g., $\mathrm{Ir} 1, \mathrm{Pt} 2)$ indicate layer thicknesses in $\mathrm{nm}$ and there are 10 stacked repeats of the $\left[\mathrm{Ir}_{1} \mathrm{Fe}_{0.5} \mathrm{Co}_{0.5} \mathrm{Pt}_{1}\right]$ unit. (c) Magnetization $M(H)$ and topological Hall resistivity $\rho^{\mathrm{TH}}(H)$ at temperature $T=5 \mathrm{~K}$ for a $\left[\operatorname{Ir}_{1} \mathrm{Fe}_{0.5} \mathrm{Co}_{0.5} \mathrm{Pt}_{1}\right]^{10}$ film. Arrows indicate field sweep directions; green/ red dashed lines indicate skyrmion nucleation/annihilation fields $H_{\text {nuc }} / H_{\mathrm{ann}}$, respectively, and the saturation magnetization $M_{s}=1.45 \mathrm{MAm}^{-1}$. (d)-(i) MFM images at $T=5.5 \mathrm{~K}$ in a bare $\left[\operatorname{Ir}_{1} \mathrm{Fe}_{0.5} \mathrm{Co}_{0.5} \mathrm{Pt}_{1}\right]^{10}$ film during a $H=0.25 \mathrm{~T} \rightarrow-0.4 \mathrm{~T}$ sweep. Scale bars are $500 \mathrm{~nm}$; color bars indicate the MFM probe resonance shift $\Delta f$ in $\mathrm{Hz}$, proportional to $m_{z}$. (j)-(l) STS images of a $25 \mathrm{~nm} \mathrm{Nb}$ film ( $S$-type structure) in (j) $H=0 \mathrm{mT}$, (k) $30 \mathrm{mT}$, and (l) $60 \mathrm{mT}$ at $T=0.4 \mathrm{~K}$. Scale bars are $100 \mathrm{~nm}$; the color bar shows the normalized zero-bias conductance (see the Supplemental Material, Sec. IV [28]) which rises inside the vortex cores.

skyrmion-antivortex exchange coupling and identify routes to optimize this effect.

Heterostructure design.-MS (chiral magnetsuperconductor) samples comprise a $\mathrm{Nb}$ layer deposited directly onto a $\left[\mathrm{Ir}_{1} \mathrm{Fe}_{0.5} \mathrm{Co}_{0.5} \mathrm{Pt}_{1}\right]^{10}$ film [schematized in Fig. 1(b)], whereas MIS (chiral magnet-insulator-superconductor) samples include a $5 \mathrm{~nm}$ insulating $\mathrm{MgO}$ layer to suppress exchange coupling. Fabrication details are in Sec. I of the Supplemental Material [28].

Magnetic layer.-Our selection of $\left[\mathrm{Ir}_{1} \mathrm{Fe}_{0.5} \mathrm{Co}_{0.5} \mathrm{Pt}_{1}\right]^{10}$ is explained in the Supplemental Material, Sec. II A [28] and its magnetic properties are summarized in Fig. 1(c). Upon reducing the field from ferromagnetic saturation, a drop in the magnetization $M(H)$ coincides with a rise in the topological Hall resistivity $\rho^{\mathrm{TH}}(H)$, signifying skyrmion nucleation at $H_{\text {nuc }}$. Magnetic force microscopy (MFM) images acquired in downward field sweeps [Figs. 1(d)-1(i)] reveal isolated skyrmions at fields close to the peaks in $\rho^{\mathrm{TH}}(H)$ [Fig. 1(e)]. At lower fields, the skyrmions proliferate and coalesce into wormlike structures [Fig. 1(f)], merging into labyrinthine stripes close to zero field [Fig. 1(g)]. After field reversal, stripes eventually split into individual skyrmions [Fig. 1(h)] with a concomitant peak in $\rho^{\mathrm{TH}}(H)$, prior to erasure at $H_{\mathrm{ann}}$ in agreement with previous studies [60-62]. MFM image analysis (see the Supplemental Material, Sec. II B [28]) indicates a typical $r_{\mathrm{sk}}=51 \pm 6 \mathrm{~nm}$.

Superconducting layer.-Knowing $r_{\mathrm{sk}}$, we can tune the $\mathrm{Nb}$ layer thickness to optimize skyrmion-vortex coupling. In films of thickness $d_{s}<\lambda$, Meissner screening is weak and $\lambda$ is replaced by the Pearl depth $\Lambda=\lambda^{2} / d_{s}$ [63]. To ensure long-range vortex interactions and negligible $\mathrm{Nb}$ bulk pinning, we select $d_{s}=25 \mathrm{~nm}$, resulting in $\Lambda \gtrsim$ $200 \mathrm{~nm}$ (see the Supplemental Material, Sec. III [28]). Figures 1(j)-1(l) display the low-field vortex matter in a $25 \mathrm{~nm} \mathrm{Nb}$ film with $T_{c}=6.05 \mathrm{~K}$, imaged by scanning tunneling spectroscopy (STS). The spatial evolution of the zero-bias conductance suggests a coherence length $\xi_{\text {eff }}(0.4 \mathrm{~K}) \approx 36 \mathrm{~nm}$ (see the Supplemental Material, Sec. IV [28]), far exceeding the Ginzburg-Landau (GL) $\xi(0) \approx 10 \mathrm{~nm}$ (see the Supplemental Material, Sec. III [28]). This disparity likely originates from vortex core expansion in the $\mathrm{Pt}$ layer encapsulating the $\mathrm{Nb}$ against oxidation [64]. Our heterostructures thus satisfy $\xi<\xi_{\text {eff }}<r_{\text {sk }}<\Lambda$.

Antivortices induced by skyrmions.-Figure 2(a) shows the total moment $m(H)$ of a MS heterostructure. Above $T_{c}$, $m(H)$ displays the expected hysteresis from the magnetic layer; below $T_{c}$ superconductivity dominates. A MIS heterostructure [Fig. 2(b)] exhibits similar behavior. In 
Fig. 2(c), we isolate the superconducting component $M_{\text {super }}(H)$ and track its evolution while decreasing $H$. Below $H_{\text {nuc }}$, the chiral film minimizes its free energy by nucleating (negatively magnetized) skyrmionic domains. This coincides with $M_{\text {super }}(H)$ turning sharply toward increasingly negative values in both MS and MIS samples. In contrast, $M_{\text {super }}(H)$ of a bare $25 \mathrm{~nm} \mathrm{Nb}$ film $(S)$ evolves smoothly through this field range. The change in $M_{\text {super }}$ below $H_{\text {nuc }}$ indicates two processes in our heterostructures: (1) ejection of vortices (with moment $m>0$ ) and (2) antivortex formation with $m<0$ (parallel to the skyrmion cores).

The saturation magnetization of our chiral film $\mu_{0} M_{s}=1.82 \mathrm{~T}$ exceeds the lower critical field of the $\mathrm{Nb}$ film $H_{c 1} \approx 0.012 \mathrm{~T}$ at $2 \mathrm{~K}$ (see the Supplemental Material,
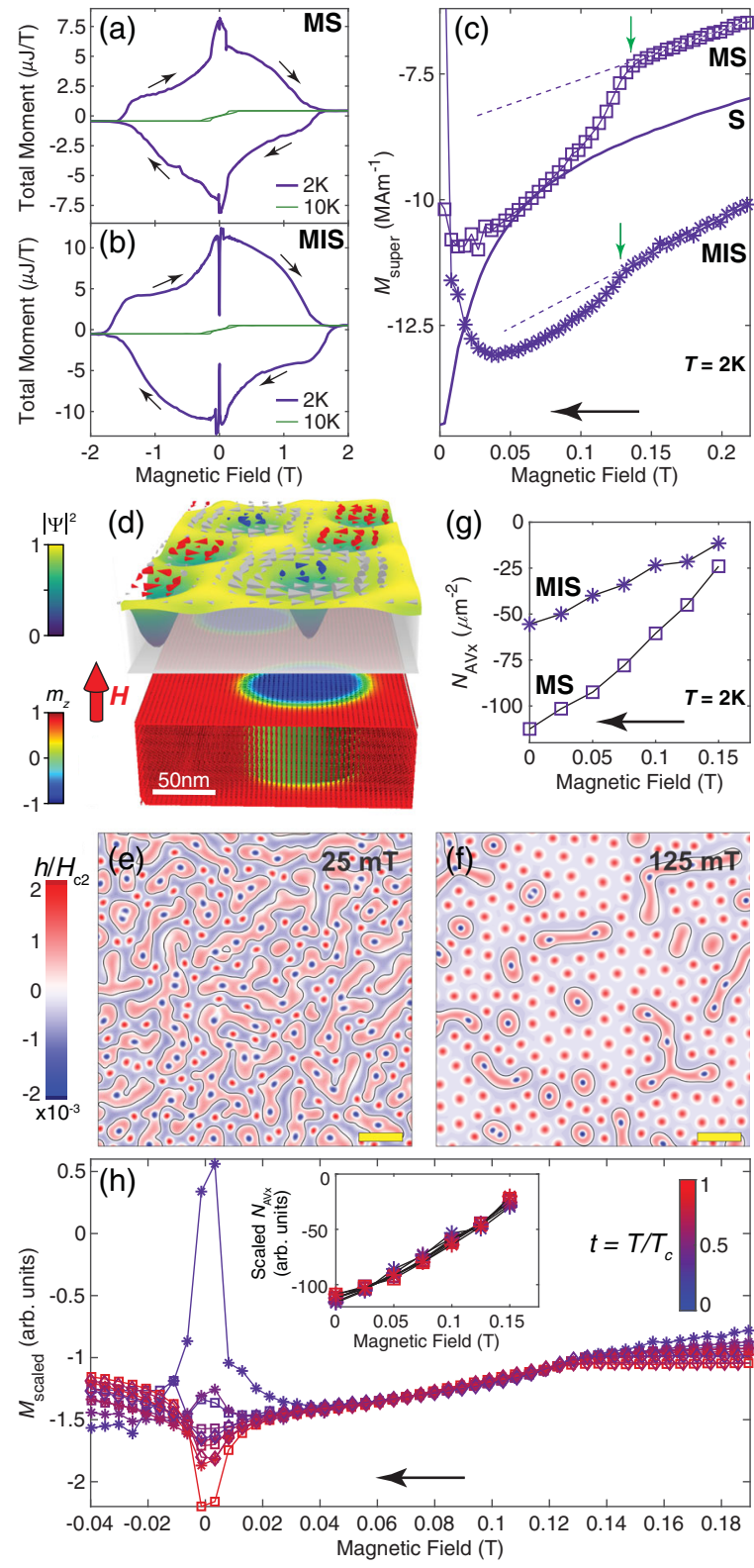

FIG. 2. Continues on facing page.
Sec. III [28]) [65], indicating that magnetic textures may form (anti)vortices in the superconductor [3]. Spontaneous vortex formation is well established in superconductor-ferromagnet hybrids [66-69], but far more challenging to achieve using chiral magnets since $r_{\mathrm{sk}}$ can be orders of magnitude smaller than typical ferromagnetic domains. Modelling the radial spin evolution as $m_{z} \sim \tanh \left[\pi\left(r-r_{\mathrm{sk}}\right) / r_{\mathrm{sk}}\right]$ [70], we estimate an upper limit of $-4.44 \Phi_{0}$ for the flux through our $50 \mathrm{~nm}$ Néel skyrmions. Although the skyrmion stray field decays rapidly outside the magnetic layer, the flux piercing the adjacent superconductor remains sufficient to create antivortices in all our heterostructures (see the Supplemental Material, Sec. VI A [28]). A threshold $M_{s} \geq \Phi_{0} \ln (\Lambda / \xi) /\left(0.86 \pi^{2} d_{m} r_{\text {sk }}\right)$ was derived in Ref. [4] for vortex formation by zero-field skyrmions, which our heterostructures exceed by $34 \%$ at $2 \mathrm{~K}$. As a definite proof of antivortex nucleation in our experiments, we combine micromagnetic simulations of the $\left[\mathrm{Ir}_{1} \mathrm{Fe}_{0.5} \mathrm{Co}_{0.5} \mathrm{Pt}_{1}\right]^{10}$ stray field (see the Supplemental Material, Sec. VI A [28]) with GL simulations of the superfluid $\left(|\Psi|^{2}\right)$ and supercurrent densities in an adjacent $25 \mathrm{~nm} \mathrm{Nb}$ film (see the Supplemental Material, Secs. VI B and C [28]). Figure 2(d) shows the supercurrent and $|\Psi|^{2}$ profiles above two Néel skyrmions stabilized at $H=125 \mathrm{mT}$, confirming the coexistence of skyrmion-induced antivortices (blue arrows) with vortices parallel to $H$ (red arrows), separated by screening currents (gray arrows) above the skyrmion domain walls. In Figs. 2(e) and 2(f), we present large simulations of vortex-antivortex states at $H=25,125 \mathrm{mT}$, visualized using the supercurrentinduced field $h$. At all fields, skyrmions and stripe domains

FIG. 2. Magnetization loops at $2 \mathrm{~K}, 10 \mathrm{~K}$ for (a) a MS sample $\left(T_{c}=5.75 \mathrm{~K}\right)$ and (b) a MIS sample $\left(T_{c}=6.25 \mathrm{~K}\right)$. Black arrows indicate field sweep directions. (c) Evolution of the superconducting magnetization $M_{\text {super }}(H)$ through $H_{\text {nuc }}$, extracted by subtracting $m(H, T=10 \mathrm{~K})$ from $m\left(H, T<T_{c}\right)$. Data from a reference $25 \mathrm{~nm} \mathrm{Nb}$ film are labeled $S$. Green arrows and dashed lines highlight the change in $M_{\text {super }}(H)$ below $H_{\text {nuc }}$. For complete $T$-dependent $M_{\text {super }}(H)$ loops, see the Supplemental Material, Sec. V [28]. (d) Calculated supercurrent profile above two Néel skyrmions at $H=125 \mathrm{mT}$. Blue arrows indicate antivortex currents induced above skyrmion cores; red arrows depict vortex currents outside skyrmion domains. Vortexantivortex annihilation is prevented by supercurrents screening the skyrmion stray field (gray arrows), maximal at the skyrmion domain wall. (e),(f) Supercurrent-induced field $h$ simulated at $H=25,125 \mathrm{mT}$, where vortices/antivortices are visible as red/ blue dots and solid lines show the contours of magnetic domain walls. Scale bars are $250 \mathrm{~nm}$; simulations from $150 \rightarrow-200 \mathrm{mT}$ are shown in the Supplemental Material, Sec. VIC [28]. (g) Field-dependent simulated antivortex density $N_{\mathrm{AVx}}$ for MS and MIS heterostructures. (h) Scaled magnetization $M_{\text {scaled }}=$ $a M_{\text {super }}+b$ in MS samples (squares, diamonds) and a MIS sample (asterisks) for $2 \mathrm{~K}<T<5 \mathrm{~K}$. The inset shows a similar scaling for $N_{\mathrm{AVx}}(H)$. 
[solid lines in Figs. 2(e) and 2(f)] robustly generate antivortices.

As $H$ falls, the simulated antivortex density $N_{\mathrm{AV} \mathrm{x}}$ rises [Fig. 2(g)]. MS heterostructures develop a higher $N_{\mathrm{AVx}}$ than MIS samples, since they experience a stronger stray field (as the $\mathrm{Nb}$ layer is $7 \mathrm{~nm}$ closer to the magnetic film). This higher $N_{\mathrm{AVx}}$ is visible in experiments [Fig. 2(c)] as a larger drop in $M_{\text {super }}$ below $H_{\text {nuc }}$ for the MS sample. Raising the temperature also increases $N_{\mathrm{AVx}}$, since $H_{c 1}$ falls. For $T<T_{c}, M_{\text {super }}(H)$ in all our heterostructures collapse onto a single curve between 0.04 and $0.14 \mathrm{~T}$ following a linear $a M_{\text {super }}+b$ scaling [Fig. 2(h)]. $N_{\mathrm{AVx}}(H, T)$ can be similarly rescaled onto a single curve [Fig. 2(h) inset], confirming that antivortex nucleation driven by evolving spin topology is responsible for the magnetic response of the superconductor.

Skyrmion impact on vortex dynamics.-The critical current density $J_{c}(H)$ measured by electrical transport (see the Supplemental Material, Sec. VII [28]) is plotted in Fig. 3(a). MS and MIS heterostructures both exhibit an enhanced $J_{c}\left(H_{\text {nuc }} \rightarrow H_{\text {ann }}\right)$ relative to a bare $\mathrm{Nb}$ film. This behavior is mirrored by an emergent time-dependent magnetization [Fig. 3(b)]. $M_{\text {super }}\left(H_{\text {nuc }} \rightarrow H_{\text {ann }}\right)$ relaxes toward increasingly negative magnetizations over several minutes after every reduction in $H$, leading to a measurable drop $\Delta M_{\text {super }}$. This relaxation and the rise in $J_{c}$ are caused by the chiral magnet inducing supercurrents which impede vortex motion. Just below $H_{\text {nuc }}$, the skyrmion/antivortex density is low and the initial rise in $J_{c}(H)$ originates from long-range vortex-antivortex attraction. Reducing the field increases the skyrmion density, obliging vortices moving in a flux gradient or applied current to cross chiral domains. Vortices are repelled by skyrmion screening currents [Fig. 2(d)]; however, the presence of an antivortex reduces the barrier height, enabling vortices to cross the skyrmion/ stripe in a process analogous to Klein tunneling. Flux-flow simulations are shown in Fig. 3(c); see Sec. VID as well as videos in the Supplemental Material [28]. Since $N_{\mathrm{AVx}}$ is larger in MS heterostructures, the vortex mobility is enhanced, reducing their $J_{c}$ and $\Delta M_{\text {super }}$ relative to MIS samples at intermediate fields [gray shading in Figs. 3(a) and 3(b)]. Around zero field, a sharp maximum in $J_{c}$ and $\Delta M_{\text {super }}$ originates from strong vortex pinning by labyrinthine stripes (see the Supplemental Material, Sec. II C [71]). This phenomenon also causes the upturn and scaling failure in $M_{\text {super }}(H)$ below $0.04 \mathrm{~T}$ [Figs. 2(c) and 2(h)]. However, the $J_{c}, \Delta M_{\text {super }}$ maxima in MS heterostructures are $>50 \%$ lower than in MIS samples, confirming the crucial role of antivortices in facilitating vortex motion.

Skyrmion-vortex exchange coupling.-Our STS data [Figs. 1(j)-1(1)] (see the Supplemental Material, Sec. IV [28]) suggest $\xi_{\text {eff }}(0.4 \mathrm{~K}) \approx 36 \mathrm{~nm}$ in the $2 \mathrm{~nm}$ Pt separating $\mathrm{Nb}$ from $\left[\mathrm{Ir}_{1} \mathrm{Fe}_{0.5} \mathrm{Co}_{0.5} \mathrm{Pt}_{1}\right]^{10}$. This corresponds to $\xi_{\text {eff }}(2 \mathrm{~K}) \approx 43 \mathrm{~nm}$, close to $r_{\mathrm{sk}} \approx 50 \mathrm{~nm}$. Exchange coupling will consequently be weak, since the induced
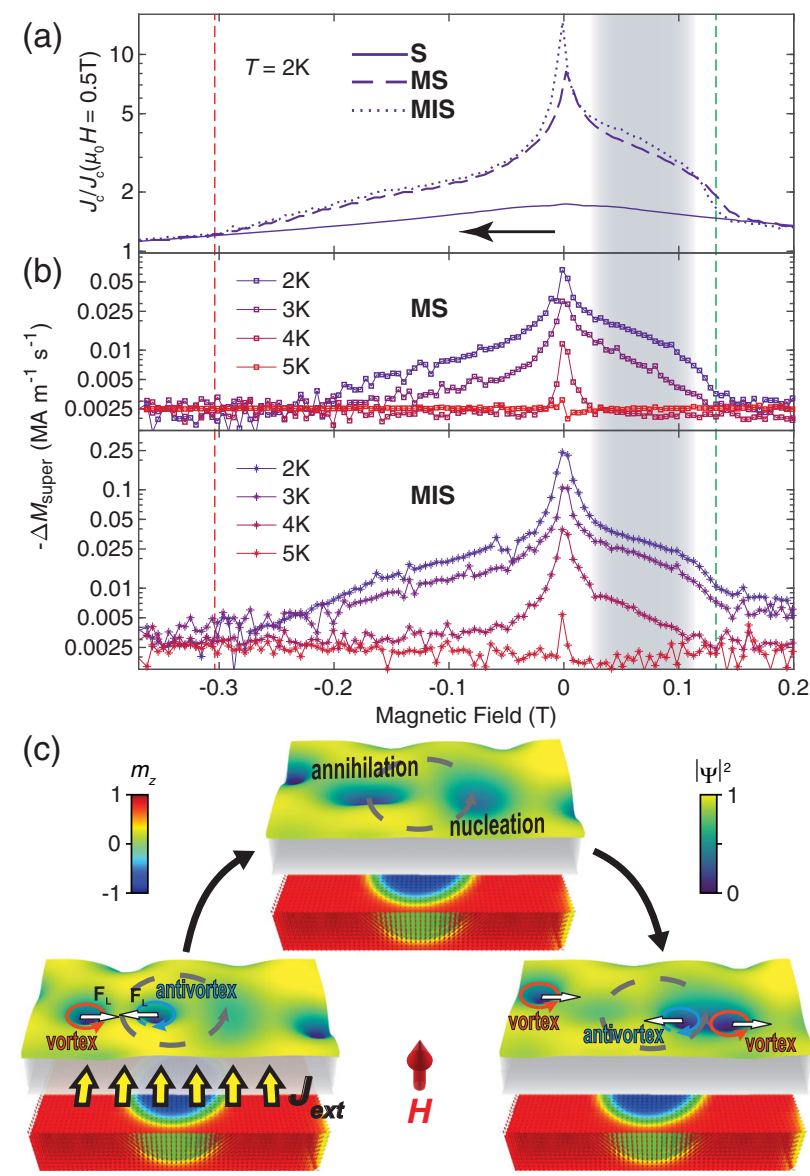

FIG. 3. (a) Field-dependent critical currents $J_{c}(H)$ in MS, MIS heterostructures and a $25 \mathrm{~nm} \mathrm{Nb}$ film $(S)$, extracted from $V(I)$ curves at $T=2 \mathrm{~K}$ (see the Supplemental Material, Sec. VII [28]). The arrow shows the field sweep direction. $H_{\text {nuc }}, H_{\text {ann }}$ from Fig. 1(c) are highlighted by green and red dashed lines. (b) Thermal evolution of the magnetic relaxation $\Delta M_{\text {super }}(H)$ in MS and MIS heterostructures. $\Delta M_{\text {super }}(H)$ is the change in $M_{\text {super }}$ in a $60 \mathrm{~s}$ period after changing the field. (c) Antivortexfacilitated vortex tunneling through chiral domains. The Lorentz force $F_{L}$ from the applied current $J_{\text {ext }}$ acts in opposite directions for vortices/antivortices. Consequently, vortex-antivortex pairs mutually annihilate on one side of the skyrmion/stripe, before spontaneously reemerging on the opposite side.

Rashba-Edelstein currents centered at $r=r_{\mathrm{sk}}$ scarcely interact with antivortex currents which peak at $r \approx 2 \xi_{\text {eff }}$. However, MFM images close to $H_{\text {nuc }}$ show larger skyrmions with $r_{\mathrm{sk}}$ up to $\sim 60 \mathrm{~nm}$ [Fig. 1(e)] (see the Supplemental Material, Sec. II B [28]). Since any increase in $r_{\text {sk }}$ should enhance exchange coupling, we search for differences between MS and MIS heterostructures at high fields. Figure 4(a) shows that the field at which $M_{\text {super }}$ first responds to skyrmion formation (denoted $H_{\mathrm{Sc}}$ ) is $\sim 20 \mathrm{mT}$ higher in MS versus MIS samples at $2 \mathrm{~K}$. This pattern is repeated in the critical current [Fig. 4(b)], where MS samples exhibit an earlier and larger $J_{c}(H)$ enhancement in the $0.16 \rightarrow 0.12 \mathrm{~T}$ field range. Since the flux lattice stray field has negligible influence on skyrmion formation (see 


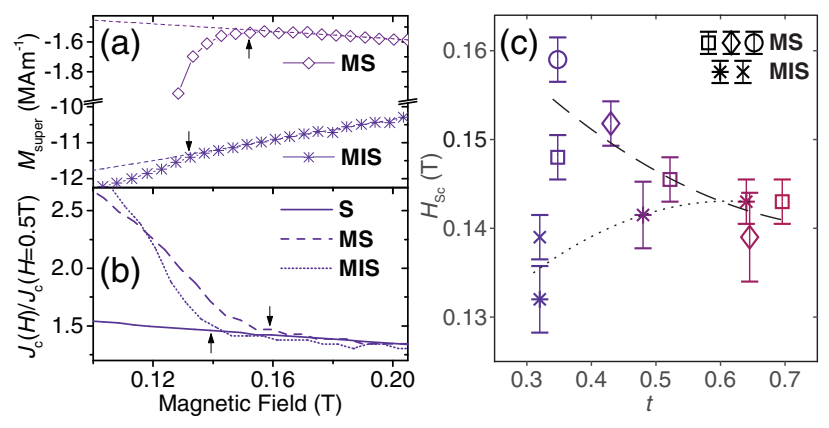

FIG. 4. (a) $M_{\text {super }}(H)$ comparison between MS and MIS samples for a downward field sweep through $H_{\text {nuc }}$ at $T=2 \mathrm{~K}$. Dashed lines are linear fits in the $0.17 \mathrm{~T}<H<$ $0.22 \mathrm{~T}$ range; arrows indicate the response field $H_{\mathrm{Sc}}$ [defined by a $1 \%$ deviation from linearity in $M_{\text {super }}(H)$ ]. (b) Comparison of normalized $J_{c}(H)$ in MS and MIS samples during a field sweep at $T=2 \mathrm{~K}$. Arrows indicate $J_{c}(H)$ rising above the background (defined by an $S$ film) at $H_{\mathrm{Sc}}$. (c) Thermal evolution of $H_{\mathrm{Sc}}$ probed by magnetization (squares, diamonds, asterisks) and transport (circle, cross); see the Supplemental Material, Sec. VIII [28] for source data and details. Dashed/dotted lines highlight the trends displayed by MS/MIS bilayers.

the Supplemental Material, Sec. IX [28]), we interpret these data as exchange coupling assisting antivortex creation at high fields, by inducing supercurrents rotating in the same sense as antivortex currents. As the temperature rises, the difference in $H_{\mathrm{Sc}}$ between MS and MIS heterostructures is suppressed [Fig. 4(c)], due to the divergence in $\xi$ which only enables the $r_{\mathrm{sk}}>\xi_{\text {eff }}$ condition to be fulfilled at low temperature. Exchange coupling can be strengthened in future hybrids by minimizing $\xi_{\text {eff }}$ at the superconductor/ chiral magnet interface.

Summary.-We have shown that skyrmion stray fields can nucleate stable antivortices in engineered chiral magnet-superconductor heterostructures. This process is independent of SOC, thus simplifying the task of building a topological superconductivity platform from (anti)vortices coupled to chiral spin textures. Coexistence of these hybrid magnetic solitons with superconducting vortices creates a complex yet controllable environment for exploring unique emergent flux dynamics.

We acknowledge support from the Singapore National Research Foundation (NRF) NRF-Investigatorship (No. NRFNRFI2015-04) and Singapore MOE Academic Research Fund Tier 3 Grant No. MOE2018-T3-1-002. M. R. thanks the National University of Singapore E6NanoFab laboratory and the Data Storage Institute, Singapore, for access to sample growth facilities. C. R. acknowledges financial support from the Swiss National Science Foundation (Grant No. 182652) and thanks A. Guipet for technical assistance. The work of R. M. M., M. J. W., and M. V. M. was supported by Research Foundation Flanders (FWO), the University of Antwerp
(BOF), and the VSC (Flemish Supercomputer Center), funded by the FWO and the Flemish GovernmentDepartment EWI. The collaboration in this work was fostered in part by EU-COST Action CA16218 NANOCOHYBRI.

*appetrovic@ntu.edu.sg

†Present address: Institute for Quantum Matter and Department of Physics and Astronomy, Johns Hopkins University, Baltimore, MD 21218, USA.

*christos@ntu.edu.sg

[1] A. N. Bogdanov and D. A. Yablonskii, Thermodynamically stable "vortices" in magnetically ordered crystals. The mixed state of magnets, Sov. Phys. JETP 68, 101 (1989), http://www.jetp.ac.ru/cgi-bin/dn/e_068_01_0101.pdf; Contribution to the theory of inhomogeneous states of magnets in the region of magnetic-field-induced phase transitions. Mixed state of antiferromagnets, Sov. Phys. JETP 69, 142 (1989), http://jetp.ac.ru/cgi-bin/dn/e_069_01_0142.pdf.

[2] K. M. D. Hals, M. Schecter, and M. S. Rudner, Composite Topological Excitations in Ferromagnet-Superconductor Heterostructures, Phys. Rev. Lett. 117, 017001 (2016).

[3] J. Baumard, J. Cayssol, F. S. Bergeret, and A. Buzdin, Generation of a superconducting vortex via Néel skyrmions, Phys. Rev. B 99, 014511 (2019).

[4] S. M. Dahir, A. F. Volkov, and I. M. Eremin, Interaction of Skyrmions and Pearl Vortices in Superconductor-Chiral Ferromagnet Heterostructures, Phys. Rev. Lett. 122, 097001 (2019).

[5] R. M. Menezes, J. F. S. Neto, C. C. S. Silva, and M. V. Milošević, Manipulation of magnetic skyrmions by superconducting vortices in ferromagnet-superconductor heterostructures, Phys. Rev. B 100, 014431 (2019).

[6] M. Garnier, A. Mesaros, and P. Simon, Topological superconductivity with orbital effects in magnetic skyrmion based heterostructures, arXiv:1909.12671.

[7] T. Yokoyama and J. Linder, Josephson effect through magnetic skyrmions, Phys. Rev. B 92, 060503(R) (2015).

[8] S. S. Pershoguba, S. Nakosai, and A. V. Balatsky, Skyrmion-induced bound states in a superconductor, Phys. Rev. B 94, 064513 (2016).

[9] S. Nakosai, Y. Tanaka, and N. Nagaosa, Two-dimensional $p$-wave superconducting states with magnetic moments on a conventional s-wave superconductor, Phys. Rev. B 88, 180503(R) (2013).

[10] W. Chen and A. P. Schnyder, Majorana edge states in superconductor-noncollinear magnet interfaces, Phys. Rev. B 92, 214502 (2015).

[11] G. Yang, P. Stano, J. Klinovaja, and D. Loss, Majorana bound states in magnetic skyrmions, Phys. Rev. B 93, 224505 (2016).

[12] U. Güngördü, S. Sandhoefner, and A. A. Kovalev, Stabilization and control of Majorana bound states with elongated skyrmions, Phys. Rev. B 97, 115136 (2018).

[13] S. Rex, I. V. Gornyi, and A. D. Mirlin, Majorana bound states in magnetic skyrmions imposed onto a superconductor, Phys. Rev. B 100, 064504 (2019).

[14] M. Garnier, A. Mesaros, and P. Simon, Topological superconductivity with deformable magnetic skyrmions, Commun. Phys. 2, 126 (2019). 
[15] T. Zhou, N. Mohanta, J. E. Han, A. Matos-Abiague, and I. Žutić, Tunable magnetic textures in spin valves: From spintronics to Majorana bound states, Phys. Rev. B 99, 134505 (2019).

[16] S. Rex, I. V. Gornyi, and A. D. Mirlin, Majorana modes in emergent-wire phases of helical and cycloidal magnet-superconductor hybrids, Phys. Rev. B 102, 224501 (2020).

[17] T. Posske, C.-K. Chiu, and M. Thorwart, Vortex Majorana braiding in a finite time, Phys. Rev. Research 2, 023205 (2020).

[18] X. Ma, C. J. O. Reichhardt, and C. Reichhardt, Braiding Majorana fermions and creating quantum logic gates with vortices on a periodic pinning structure, Phys. Rev. B 101, 024514 (2020).

[19] Y. L. Wang, X. Ma, J. Xu, Z. L. Xiao, A. Snezhko, R. Divan, L. E. Ocola, J. E. Pearson, B. Janko, and W. K. Kwok, Switchable geometric frustration in an artificial-spin-icesuperconductor heterosystem, Nat. Nanotechnol. 13, 560 (2018).

[20] V. M. Edelstein, Magnetoelectric Effect in Polar Superconductors, Phys. Rev. Lett. 75, 2004 (1995).

[21] K. M. D. Hals, Magnetoelectric coupling in superconductorhelimagnet heterostructures, Phys. Rev. B 95, 134504 (2017).

[22] R. Takashima and S. Fujimoto, Supercurrent-induced skyrmion dynamics and tunable Weyl points in chiral magnet with superconductivity, Phys. Rev. B 94, 235117 (2016).

[23] K. M. D. Hals, Supercurrent-induced spin-orbit torques, Phys. Rev. B 93, 115431 (2016).

[24] S. M. Dahir, A. F. Volkov, and I. M. Eremin, Meissner currents induced by topological magnetic textures in hybrid superconductor / ferromagnet structures, Phys. Rev. B 102, 014503 (2020).

[25] M. Hervé, B. Dupé, R. Lopes, M. Böttcher, M. D. Martins, T. Balashov, L. Gerhard, J. Sinova, and W. Wulfhekel, Stabilizing spin spirals and isolated skyrmions at low magnetic field exploiting vanishing magnetic anisotropy, Nat. Commun. 9, 1015 (2018).

[26] N. Romming, A. Kubetzka, C. Hanneken, K. von Bergmann, and R. Wiesendanger, Field-Dependent Size and Shape of Single Magnetic Skyrmions, Phys. Rev. Lett. 114, 177203 (2015).

[27] A. Kubetzka, J. M. Bürger, R. Wiesendanger, and K. von Bergmann, Towards skyrmion-superconductor hybrid systems, Phys. Rev. Mater. 4, 081401(R) (2020).

[28] See Supplemental Material http://link.aps.org/supplemental/ 10.1103/PhysRevLett.126.117205 for additional details of our heterostructure growth, experimental characterization, and theoretical analysis, including Refs. [29-59].

[29] A. Yagil, A. Almoalem, A. Soumyanarayanan, A. K. C. Tan, M. Raju, C. Panagopoulos, and O. M. Auslaender, Stray field signatures of Néel textured skyrmions in $\mathrm{Ir} / \mathrm{Fe} / \mathrm{Co} / \mathrm{Pt}$ multilayer films, Appl. Phys. Lett. 112, 192403 (2018).

[30] K. Il'in, M. Siegel, A. Semenov, A. Engel, and H. W. Hübers, Critical current of $\mathrm{Nb}$ and $\mathrm{NbN}$ thin-film structures: The cross-section dependence, Phys. Status Solidi C 2, 1680 (2005).

[31] W. Wang, M. W. Daniels, Z. Liao, Y. Zhao, J. Wang, G. Koster, G. Rijnders, C.-Z. Chang, D. Xiao, and W. Wu, Spin chirality fluctuation in two-dimensional ferromagnets with perpendicular magnetic anisotropy, Nat. Mater. 18, 1054 (2019).

[32] R. Tomasello, K. Y. Guslienko, M. Ricci, A. Giordano, J. Barker, M. Carpentieri, O. Chubykalo-Fesenko, and G. Finocchio, Origin of temperature and field dependence of magnetic skyrmion size in ultrathin nanodots, Phys. Rev. B 97, 060402(R) (2018).

[33] X. S. Wang, H. Y. Yuan, and X. R. Wang, A theory on skyrmion size, Commun. Phys. 1, 31 (2018).

[34] F. Büttner, I. Lemesh, and G. S. Beach, Theory of isolated magnetic skyrmions: From fundamentals to room temperature applications, Sci. Rep. 8, 4464 (2018).

[35] K. Harada, O. Kamimura, H. Kasai, T. Matsuda, A. Tonomura, and V. V. Moshchalkov, Direct observation of vortex dynamics in superconducting films with regular arrays of defects, Science 274, 1167 (1996).

[36] C. Reichhardt, C. J. Olson, and F. Nori, Commensurate and incommensurate vortex states in superconductors with periodic pinning arrays, Phys. Rev. B 57, 7937 (1998).

[37] I. Zaytseva, O. Abal'Oshev, P. Dłuzewski, W. Paszkowicz, L. Y. Zhu, C. L. Chien, M. Kończykowski, and M.Z. Cieplak, Negative Hall coefficient of ultrathin niobium in $\mathrm{Si} / \mathrm{Nb} / \mathrm{Si}$ trilayers, Phys. Rev. B 90, 060505(R) (2014).

[38] N. W. Ashcroft and N.D. Mermin, Solid State Physics (Harcourt College Publishers, New York, 1976), p. 757.

[39] F. E. Harper and M. Tinkham, The mixed state in superconducting thin films, Phys. Rev. 172, 441 (1968).

[40] V. G. Kogan, Parallel nucleation field in thin superconducting films, Phys. Rev. B 34, 3499 (1986).

[41] M. Trezza, S. L. Prischepa, C. Cirillo, R. Fittipaldi, M. Sarno, D. Sannino, P. Ciambelli, M. B. Hesselberth, S. K. Lazarouk, A. V. Dolbik, V. E. Borisenko, and C. Attanasio, Superconducting properties of $\mathrm{Nb}$ thin films deposited on porous silicon templates, J. Appl. Phys. 104, 083917 (2008).

[42] F. J. Blunt, A. R. Perry, A. M. Campbell, and R. S. Liu, An investigation of the appearance of positive magnetic moments on field cooling some superconductors, Physica (Amsterdam) 175C, 539 (1991).

[43] R. C. Dynes, V. Narayanamurti, and J. P. Garno, Direct Measurement of Quasiparticle-Lifetime Broadening in a Strong-Coupled Superconductor, Phys. Rev. Lett. 41, 1509 (1978).

[44] M. L. A. MacVicar, Distribution of the energy gap in $k$ space for superconducting $\mathrm{Nb}$, Phys. Rev. B 2, 97 (1970).

[45] A. Odobesko, F. Friedrich, S. B. Zhang, S. Haldar, S. Heinze, B. Trauzettel, and M. Bode, Anisotropic vortices on superconducting $\mathrm{Nb}(110)$, Phys. Rev. B 102, 174502 (2020).

[46] C. Di Giorgio, D. D’Agostino, A. M. Cucolo, M. Iavarone, A. Scarfato, G. Karapetrov, S. A. Moore, M. Polichetti, D. Mancusi, S. Pace, V. Novosad, V. Yefremenko, and F. Bobba, Superconducting vortex-antivortex pairs: Nucleation and confinement in magnetically coupled superconductor-ferromagnet hybrids, in Vortex Dynamics and Optical Vortices, edited by H. Perez-De-Tejada (IntechOpen, London, 2017), pp. 83-106.

[47] H. Alloul, Introduction to the Physics of Electrons in Solids (Springer-Verlag, Berlin, Heidelberg, 2011), p. 611. 
[48] C. Caroli, P. G. De Gennes, and J. Matricon, Bound fermion states on a vortex line in a type II superconductor, Phys. Lett. 9, 307 (1964).

[49] C. Dubois, G. Santi, I. Cuttat, C. Berthod, N. Jenkins, A. P. Petrović, A. A. Manuel, and Ø. Fischer, Scanning Tunneling Spectroscopy in the Superconducting State and Vortex Cores of the $\beta$-Pyrochlore $\mathrm{KOs}_{2} \mathrm{O}_{6}$, Phys. Rev. Lett. 101, 057004 (2008).

[50] A. Vansteenkiste, J. Leliaert, M. Dvornik, F. GarciaSanchez, and B. Van Waeyenberge, The design and verification of Mumax3, AIP Adv. 4, 107133 (2014).

[51] J. Leliaert, M. Dvornik, J. Mulkers, J. De Clercq, M. V. Milošević, and B. Van Waeyenberge, Fast micromagnetic simulations on GPU-Recent advances made with mumax3, J. Phys. D 51, 123002 (2018).

[52] L. Embon, Y. Anahory, Z. L. Jelić, E. O. Lachman, Y. Myasoedov, M. E. Huber, G. P. Mikitik, A. V. Silhanek, M. V. Milošević, A. Gurevich, and E. Zeldov, Imaging of super-fast dynamics and flow instabilities of superconducting vortices, Nat. Commun. 8, 85 (2017).

[53] L. Kramer and R. J. Watts-Tobin, Theory of Dissipative Current-Carrying States in Superconducting Filaments, Phys. Rev. Lett. 40, 1041 (1978).

[54] N. Kopnin, Theory of Nonequilibrium Superconductivity (Clarendon Press, Oxford, 2001).

[55] N. G. Ptitsina, G. M. Chulkova, K. S. Il'in, A. V. Sergeev, F. S. Pochinkov, E. M. Gershenzon, and M. E. Gershenson, Electron-phonon interaction in disordered metal films: The resistivity and electron dephasing rate, Phys. Rev. B 56, 10089 (1997).

[56] M. V. Milošević, S. V. Yampolskii, and F. M. Peeters, Magnetic pinning of vortices in a superconducting film: The (anti)vortex-magnetic dipole interaction energy in the London approximation, Phys. Rev. B 66, 174519 (2002).

[57] A. Shekhter, L. N. Bulaevskii, and C. D. Batista, Vortex Viscosity in Magnetic Superconductors due to Radiation of Spin Waves, Phys. Rev. Lett. 106, 037001 (2011).

[58] A. A. Bespalov, A. S. Mel'nikov, and A. I. Buzdin, Magnon radiation by moving Abrikosov vortices in ferromagnetic superconductors and superconductor-ferromagnet multilayers, Phys. Rev. B 89, 054516 (2014).

[59] A. Angrisani Armenio, C. Bell, J. Aarts, and C. Attanasio, High-velocity instabilities in the vortex lattice of $\mathrm{Nb}$ / permalloy bilayers, Phys. Rev. B 76, 054502 (2007).

[60] A. Soumyanarayanan, M. Raju, A. L. Gonzalez Oyarce, A. K. C. Tan, M. Y. Im, A. P. Petrović, P. Ho, K. H. Khoo, M. Tran, C. K. Gan, F. Ernult, and C. Panagopoulos, Tunable room-temperature magnetic skyrmions in $\mathrm{Ir} / \mathrm{Fe} / \mathrm{Co} / \mathrm{Pt}$ multilayers, Nat. Mater. 16, 898 (2017).
[61] M. Raju, A. Yagil, A. Soumyanarayanan, A. K. C. Tan, A. Almoalem, O. M. Auslaender, and C. Panagopoulos, Evolution of chiral magnetic textures and their topological Hall signature in $\mathrm{Ir} / \mathrm{Fe} / \mathrm{Co} / \mathrm{Pt}$ multilayer films, Nat. Commun. 10, 696 (2019).

[62] N. K. Duong, M. Raju, A. P. Petrović, R. Tomasello, G. Finocchio, and C. Panagopoulos, Stabilizing zero-field skyrmions in $\mathrm{Ir} / \mathrm{Fe} / \mathrm{Co} / \mathrm{Pt}$ thin film multilayers by magnetic history control, Appl. Phys. Lett. 114, 072401 (2019).

[63] J. Pearl, Current Distribution in Superconducting Films Carrying Quantized Fluxoids, Appl. Phys. Lett. 5, 65 (1964).

[64] V. S. Stolyarov, T. Cren, C. Brun, I. A. Golovchanskiy, O. V. Skryabina, D. I. Kasatonov, M. M. Khapaev, M. Y. Kupriyanov, A. A. Golubov, and D. Roditchev, Expansion of a superconducting vortex core into a diffusive metal, Nat. Commun. 9, 2277 (2018).

[65] N. Pinto, S. J. Rezvani, A. Perali, L. Flammia, M. V. Milošević, M. Fretto, C. Cassiago, and N. De Leo, Dimensional crossover and incipient quantum size effects in superconducting niobium nanofilms, Sci. Rep. 8, 4710 (2018).

[66] I. F. Lyuksyutov and V. L. Pokrovsky, Ferromagnet-superconductor hybrids, Adv. Phys. 54, 67 (2005).

[67] A. Y. Aladyshkin, A. V. Silhanek, W. Gillijns, and V. V. Moshchalkov, Nucleation of superconductivity and vortex matter in superconductor-ferromagnet hybrids, Supercond. Sci. Technol. 22, 053001 (2009).

[68] M. Iavarone, A. Scarfato, F. Bobba, M. Longobardi, G. Karapetrov, V. Novosad, V. Yefremenko, F. Giubileo, and A. M. Cucolo, Imaging the spontaneous formation of vortex-antivortex pairs in planar superconductor/ ferromagnet hybrid structures, Phys. Rev. B 84, 024506 (2011).

[69] F. Bobba, C. Di Giorgio, A. Scarfato, M. Longobardi, M. Iavarone, S. A. Moore, G. Karapetrov, V. Novosad, V. Yefremenko, and A. M. Cucolo, Vortex-antivortex coexistence in Nb-based superconductor/ferromagnet heterostructures, Phys. Rev. B 89, 214502 (2014).

[70] M. Heide, G. Bihlmayer, and S. Blügel, DzyaloshinskiiMoriya interaction accounting for the orientation of magnetic domains in ultrathin films: Fe/W(110), Phys. Rev. B 78, 140403(R) (2008).

[71] X. Palermo, N. Reyren, S. Mesoraca, A. V. Samokhvalov, S. Collin, F. Godel, A. Sander, K. Bouzehouane, J. Santamaria, V. Cros, A. I. Buzdin, and J. E. Villegas, Tailored Flux Pinning in Superconductor-Ferromagnet Multilayers with Engineered Magnetic Domain Morphology From Stripes to Skyrmions, Phys. Rev. Applied 13, 014043 (2020). 